\title{
Binary Classifier Inspired by Quantum Theory
}

\author{
Prayag Tiwari \\ Department of Information Engineering \\ University of Padova \\ Via Gradenigo 6/b 35131 - Padova, Italy \\ https://sites.google.com/view/prayag-tiwari/home \\ tiwari@dei.unipd.it
}

\author{
Massimo Melucci \\ Department of Information Engineering \\ University of Padova \\ Via Gradenigo 6/b 35131 - Padova, Italy \\ http://www.dei.unipd.it/ melo/ \\ melo@dei.unipd.it
}

\begin{abstract}
Machine Learning (ML) helps us to recognize patterns from raw data. ML is used in numerous domains i.e. biomedical, agricultural, food technology, etc. Despite recent technological advancements, there is still room for substantial improvement in prediction. Current ML models are based on classical theories of probability and statistics, which can now be replaced by Quantum Theory (QT) with the aim of improving the effectiveness of ML. In this paper, we propose the Binary Classifier Inspired by Quantum Theory (BCIQT) model, which outperforms the state of the art classification in terms of recall for every category.
\end{abstract}

\section{Introduction}

ML is a set of models which can automatically identify the hidden patterns in the data and can then utilize hidden patterns to make decisions in condition of uncertainty. ML has been progressively implemented in several areas including chemistry, biomedical science and robotics. ML falls into three categories, i.e. supervised learning (e.g. classification), unsupervised learning (e.g. clustering) and reinforcement learning. In this paper we focus on classification, which is the way to represent and allocate objects into different categories.

QT is the probabilistic approach to representing and predicting properties of microscopic phenomena. Given an observable and an arbitrary state of a microscopic particle, QT computes a probability distribution of the values of the observable. The quantum formalism is explicitly acceptable to explain distinct types of stochastic processes. Several nonstandard implementations of the quantum formalism has emerged. For instance, the quantum formalism have been utilized vastly in the economic processes, game theory and cognitive science as well.

Since the data is growing exponentially, current state-ofthe-art models are still not effective. In particular, recall is still unsatisfactory because most classification models aim to maximize precision especially when the items of a class can be ranked by a certain measure of membership to the class; a glaring example is the search of the Internet. In contrast,

Copyright (C) 2019, Association for the Advancement of Artificial Intelligence (www.aaai.org). All rights reserved. recall is crucial in many daily tasks aiming to find all the pertinent items of a class such as patent search and biomedical image classification.

Our approach is to develop a new theoretical approach inspired by Quantum Mechanics (QM) in order to dig into the quantum world and come up with new and effective models which are capable of increasing recall. Our hypothesis is that, since QM has already shown its effectiveness in several fields, it may also be effective in ML. To this end we will exploit Quantum Probability theory, which is the quantum generalization of classical probability theory and was developed by Von Neumann. While classical probability theory provides that a system can be in either state 0 or 1 , quantum probability comes into existence to go beyond classical theory and describes states which can be anything in-between 0 and 1 . In this paper, we propose the BCIQT model which is a step towards shifting from classical models to quantum models.

\section{Proposed Methodology}

\section{Classical and Quantum Signal Detection Theory}

BCIQT is based on the overlap between Signal Detection Theory (SDT) and QM. The main difference between the classical framework and the quantum framework of signal detection regards what encoders encode and what decoders decode (Helstrom 1969).

In the classical framework, there is c-c (classicalclassical) mapping from a symbol to the wave to the corrupted channel; then the decoder produce c-c (classicalclassical) mapping from the corrupted channel wave to a symbol. In the quantum framework, there is a coder between the source and the channel; the classical symbol is transmitted through the quantum state. Initial encoding starts like $\mathrm{c}-\mathrm{q}$ (classical-quantum) mapping from the symbol to the quantum state selected from a finite set of possible states. More details about classical and quantum SDT can be found in (Helstrom 1969).

\section{Binary Classifier Inspired by Quantum Theory}

A novel BCIQT that is inspired by quantum detection theory is described in this section. For each category we supposed that each training sample was about the category or not. For a given category and the set of training samples, we 
used the projector $\Delta$ for each category to identify whether the test sample was about the category or not. To determine whether the test sample was about the category, $\Delta$ was examined against a vectorial representation of the test samples.

Consider a set of distinct features calculated from the whole sample collection. Each sample could be represented as a vector of features; each element in the feature vector was a non-negative number such as frequency. Each sample in the training set had a binary label in $\{0,1\}$. The main goal of BCIQT was to obtain one binary label for each sample in the test set.

The BCIQT estimated two density operators $\rho_{0}$ and $\rho_{1}$, one operator for each category or class and its complement, by using the training samples; in particular, for each class, the negative training samples were utilized to estimate $\rho_{0}$ and the positive training samples were utilized to estimate $\rho_{1}$.

In order to achieve these density operators $\rho_{0}$ and $\rho_{1}$, we first calculated the total number of samples with non-zero values for each particular feature. In such a way, one vector $|v\rangle$ was obtained for each class. Since we were considering the binary case, two vectors $\left|v_{0}\right\rangle$ and $\left|v_{1}\right\rangle$ were obtained; the former referred to the negative training samples and the latter referred to the positive training samples; these vectors may be considered as statistics of the features in a class. We normalized the vectors to obtain $|\langle v \mid v\rangle|^{2}=1$. Then, we calculated the outer product in order to obtain the density operators $\rho_{0}$ and $\rho_{1}$ as follows:

$$
\rho_{0}=\frac{\left|v_{0}\right\rangle\left\langle v_{0}\right|}{\operatorname{tr}\left(\left|v_{0}\right\rangle\left\langle v_{0}\right|\right)} \quad \rho_{1}=\frac{\left|v_{1}\right\rangle\left\langle v_{1}\right|}{\operatorname{tr}\left(\left|v_{1}\right\rangle\left\langle v_{1}\right|\right)}
$$

We computed the projection operator $\Delta$ according to (Melucci 2016), that is,

$$
\rho_{1}-\lambda \rho_{0}=\eta \Delta+\beta \Delta^{\perp} \quad \eta>0 \quad \beta<0 \quad \Delta \Delta^{\perp}=0
$$

where $\xi$ is the prior probability of the negative class and $\lambda=\xi /(1-\xi)$; moreover, $\eta$ is the positive eigenvalue corresponding to $\Delta$ which represents the subspaces of the vectors representing the sample to be accepted in the target class.

We set $\lambda=1$ to simply mean that both classes had the same prior probability $(\xi=0.5)$; moreover, there was no cost for wrong detection $C_{00}=C_{11}=0$; finally, the costs of false alarm and miss were constant $\left(C_{01}=C_{10}\right)$. Eventually, we determined the binary label for the given test sample $S_{j}$ by inspecting the value of $\left\langle w_{S_{j}}|\Delta| w_{S_{j}}\right\rangle$ : If $\left\langle w_{S_{j}}|\Delta| w_{S_{j}}\right\rangle \geq$ 0.5 , then $C\left(S_{j}\right)=1$; otherwise $C\left(S_{j}\right)=0$.

\section{Experiment}

The MNIST database ${ }^{1}$ of handwritten digits has a training set of 60,000 examples, and a test set of 10,000 examples. There are 9 categories from 0 to 9 but excluded 9 . It is a subset of a larger set available from the National Institute of Standards and Technology (NIST). The digits have been size-normalized and centered in a fixed-size image.

The four models i.e. Naïve Bayes (NB), Support Vector Machine (SVM), $k$ Nearest Neighbours (k-NN) and Decision Tree (DT) were used as baselines. Prior to training the models, the top 100 features were selected as the best features for all the models in terms of recall. The chi-square feature selection model was used.

We used one-vs-all strategy: for each category, the training samples labeled as pertinent to the category are considered positive examples, while the rest are considered negative examples. While training the model, five fold cross validation was used. As it can be seen from Table 1, our proposed model performs better than any state-of-art-model in terms of recall for every category. By changing number of features, evaluation measures(i.e. accuracy, precision, recall and f-measure) also change and provide comparable results to the baselines.

Table 1: Comparison of Recall among k-nearest neighbors(KNN), Decision Tree(DT), Naive Bayes (NB), Support Vector Machine (SVM) and Binary Classifier Inspired by Quantum Theory (BCIQT)

\begin{tabular}{cccccc}
\hline Category & KNN & DT & NB & SVM & BCIQT \\
\hline 0 & 0.959 & 0.884 & 0.889 & 0.292 & $\mathbf{1}$ \\
1 & 0.699 & 0.710 & 0.582 & 0.390 & $\mathbf{0 . 9 9 6}$ \\
2 & 0.704 & 0.652 & 0.709 & 0.474 & $\mathbf{1}$ \\
3 & 0.623 & 0.508 & 0.792 & 0.346 & $\mathbf{0 . 9 9 7}$ \\
4 & 0.643 & 0.621 & 0.666 & 0.259 & $\mathbf{0 . 9 9 9}$ \\
5 & 0.892 & 0.855 & 0.872 & 0.621 & $\mathbf{1}$ \\
6 & 0.743 & 0.755 & 0.873 & 0.454 & $\mathbf{0 . 9 9 9}$ \\
7 & 0.753 & 0.728 & 0.779 & 0.332 & $\mathbf{1}$ \\
8 & 0.749 & 0.677 & 0.817 & 0.209 & $\mathbf{1}$ \\
\hline
\end{tabular}

\section{Conclusion and Future Works}

We found out that our proposed model outperforms the stateof-the-art models in terms of recall; therefore, this model can be safely implemented if someone is looking for high recall. We believe that this is an encouraging result and opens a gateway towards quantum inspired ML approaches. As for future work, we would like to develop multi-class classifiers (i.e. how to assign an item to more than one class) and multilabel classifiers (i.e. how to deal with non-binary labels), and re-rank the test items of a class by increasing precision as well.

\section{Acknowledgments}

"This project has received funding from the European Union's Horizon 2020 research and innovation programme under the Marie Sklodowska-Curie grant agreement No $721321 "$.

\section{References}

Helstrom, C. W. 1969. Quantum detection and estimation theory. Journal of Statistical Physics 1(2):231-252.

Melucci, M. 2016. Relevance feedback algorithms inspired by quantum detection. IEEE TKDE 28(4):1022-1034.

\footnotetext{
${ }^{1}$ http://yann.lecun.com/exdb/mnist/
} 